\title{
O.S.P.
}

L'orientation scolaire et professionnelle

$43 / 3$ | 2014

Varia

\section{Les familles à l'épreuve des ruptures scolaires}

Families under stress of school expulsion

\section{Michel Tondellier}

\section{(2) OpenEdition}

Journals

Édition électronique

URL : http://journals.openedition.org/osp/4421

DOI : 10.4000/osp.4421

ISSN : 2104-3795

Éditeur

Institut national d'étude du travail et d'orientation professionnelle (INETOP)

Édition imprimée

Date de publication : 15 septembre 2014

ISSN : 0249-6739

Référence électronique

Michel Tondellier, "Les familles à l'épreuve des ruptures scolaires », L'orientation scolaire et professionnelle [En ligne], 43/3 | 2014, mis en ligne le 15 septembre 2017, consulté le 16 décembre 2020. URL : http://journals.openedition.org/osp/4421 ; DOl : https://doi.org/10.4000/osp.4421

Ce document a été généré automatiquement le 16 décembre 2020.

(c) Tous droits réservés 


\title{
Les familles à l'épreuve des ruptures scolaires
}

Families under stress of school expulsion

\author{
Michel Tondellier
}

1 En France, l'exclusion définitive des établissements scolaires ne s'est constituée que récemment en objet de recherche et restait à la périphérie d'études plus nourries sur les phénomènes de déscolarisation ${ }^{1}$. Les premières publications consacrées à ce sujet portaient sur les modalités de convocation des conseils de discipline et leur déroulement (Chevit, 2003 ; Tondellier, 2006) ou sur la fonction de cette instance dans l'organisation scolaire (Geay, Oria, \& Fromard, 2009). L'étude menée par Fotinos et Horenstein (2011) apporte sur le sujet les éclairages statistiques qui lui faisaient jusqu'à présent défaut. Chaque jour, 95 élèves sont exclus définitivement d'un établissement scolaire en France, ils sont 17000 par an. 11 faut bien sûr s'interroger sur les causes et significations de l'inégale distribution de ces expériences dans la population qui touchent les professions et catégories socioprofessionnelles (PCS) défavorisées et les établissements concernés par la politique d'éducation prioritaire ${ }^{2}$. Mais, au-delà de cette perspective institutionnelle, la question qui se pose est celle du devenir de ces élèves, dont 1'effectif correspond à celui du nombre total d'inscrits dans le secondaire public et privé dans des départements faiblement peuplés comme ceux de la Corrèze, la Meuse, l'Indre ou l'Aveyron (MEN, 2012). Que deviennent ces dizaines de milliers d'élèves? Aucune étude ne nous renseigne, par exemple, sur le taux de rescolarisation réussie, au sens où l'exclusion d'un établissement ne se solderait pas par une déscolarisation ou une sortie sans diplôme plus ou moins rapide du système éducatif français.

2 Contrairement aux travaux anglo-saxons déjà nombreux et parfois anciens ${ }^{3}$ ces premiers travaux francophones se sont concentrés sur le caractère institutionnel de 1'objet et n'ont guère abordé le caractère subjectif de 1'épreuve du point de vue des usagers. Pourtant, l'ampleur statistique du phénomène ne doit pas masquer la diversité des vécus de cette expérience ${ }^{4}$. Les exclusions définitives d'établissements scolaires, qu'ils soient publics ou privés, impliquent en effet une expérience spécifique, celle de 
l'ostracisme et des formes de «morts sociales» qui l'accompagnent (Goffman, 1952/1989). En effet, la rupture imposée avec les pairs ainsi que les tensions créées au sein de la famille (Wright, Standen, John, German, \& Patel, 2005) sont autant d'expériences qui travaillent ce que Castel appelle les « conditions de réalisation» du sujet (Castel \& Haroche, 2001), tant cette expérience pourra avoir des répercussions sur son insertion professionnelle (comme conséquence d'une désaffiliation scolaire) et sur la qualité de ses «supports » familiaux (comme conséquence d'une rupture familiale). En ce sens, l'exclusion définitive d'un établissement scolaire opère comme un révélateur ou un amplificateur de la fragilité relationnelle de 1'élève. En nous penchant plus particulièrement sur la façon dont la famille du jeune exclu accuse l'impact de l'exclusion définitive de l'établissement, nous analyserons l'importance des ressources sur lesquelles l'élève concerné peut s'appuyer et les conséquences de l'exclusion dans les situations où ces ressources font défaut. Au final, en mobilisant les travaux de Castel (1991) et ceux de Blyth et Milner (1993), l'expérience de l'exclusion définitive d'un établissement scolaire sera appelée à être replacée dans le contexte plus large de l'exclusion sociale et de l'exercice de la citoyenneté.

\section{Méthode et « spécificités » du contexte antillais}

3 La recherche repose sur l'analyse de 30 entretiens semi-directifs menés avec des individus ayant connu un passage en conseil de discipline (CDD) et l'exclusion définitive d'un établissement scolaire en Martinique. Pour négocier ces entretiens, les réseaux de connaissances interpersonnelles d'étudiants de l'Université des Antilles et de la Guyane (UAG) ont été mobilisés 5 . Le guide d'entretien commun à tous les enquêteurs a assuré une homogénéité thématique dans le recueil de données. Les entretiens ont duré entre 35 minutes et deux heures. Les informateurs sont âgés de 12 à 41 ans et d'origine sociale relativement modeste (parents petits commerçants, agriculteurs, employés de commerce, petits fonctionnaires, chômeurs).

4 La question de l'autorité parentale est révélatrice d'une première forme de fragilité relationnelle des jeunes exclus puisque dans 13 cas sur les 27 renseignés, les parents étaient séparés ou divorcés lors de l'exclusion. Lorsque l'on demande aux informateurs : qui exerçait l'autorité parentale au moment de l'exclusion? Dans les deux tiers des cas, celle-ci est exercée par une seule personne (22 sur 30). Pour 16 participants, l'autorité parentale est exercée par la seule mère (parfois aidée par une tante ou la marraine de l'enfant), plus marginalement, c'est le père seul qui l'exerce ( 3 cas). Elle est partagée par les deux parents pour 8 interviewés et, dans le reste des cas, par les grands-parents ou d'autres membres de la famille. L'échantillon compte des informateurs des deux sexes ( 25 hommes, 5 femmes). Les exclusions définitives de l'établissement scolaire concernent des élèves de la $6^{\mathrm{e}}$ à la terminale. Pour quatre des six cas exclus à plusieurs reprises, l'exclusion définitive d'un deuxième établissement se déroule l'année suivant la première exclusion et témoigne de l'échec d'intégration de l'élève dans son nouvel établissement.

5 Les indicateurs sociaux et scolaires concernant la région Martinique sont particulièrement contrastés (MEN, 2014) : le taux de retard à l'entrée en $6^{\mathrm{e}}$ est de $12,9 \%$ contre $11,4 \%$ au niveau national, mais inférieur à l'académie de Lille (13,4\%); le taux de retard en seconde est de $21 \%$, l'équivalent de Créteil pour un taux national à $17 \%$; $31,9 \%$ des jeunes sont en difficulté de lecture, le triple du taux moyen national (9,6\%).

L'orientation scolaire et professionnelle, 43/3 | 2014 
Les chiffres du taux d'insertion professionnelle 7 mois après l'obtention du diplôme témoignent de l'étroitesse du marché de l'emploi local : 8,5\% d'insertion pour les CAPBEP pour une moyenne nationale de $32,4 \% ; 28,1 \%$ pour les BTS pour une moyenne nationale de $61,4 \%$. La part des enfants dont le parent de référence est au chômage de longue durée est de $16,8 \%$ contre $4,2 \%$ au niveau national. Les statistiques isolent une spécificité profonde des structures familiales martiniquaises : en effet, plus du tiers des enfants de l'île vivent de 0 à 10 ans dans une famille monoparentale (contre un enfant sur 50 en France métropolitaine), la situation perdure jusqu'à leurs 15 ans pour $28 \%$ des enfants du département (Valentin-Marie et al., 2012).

6 Les départements d'outre-mer sont trop souvent réduits à des "spécificités » forgées par une relation de dépendance à la "métropole ", parfois marquée historiquement comme c'est le cas ici -par l'économie de la plantation et la décolonisation. L'exposé et la discussion de la nature et des modalités d'influence de ces spécificités sur le phénomène étudié dépassent très largement la problématique de cet article, mais il est postulé que ces spécificités historiques, géographiques, migratoires ou langagières ne déterminent pas à elles seules l'expérience de l'exclusion définitive d'un établissement scolaire ${ }^{6}$. Au-delà de ces spécificités qui alimentent le sens commun des discours sur l'outre-mer, il est plus heuristique d'analyser les points d'homologie de ce territoire ultramarin avec d'autres départements français ou des espaces étrangers ${ }^{7}$. Il est donc supposé que le schéma général des réactions parentales est comparable à celui de la métropole, mais que les spécificités locales lui donnent à différents égards des aspects qui, bien que particuliers, peuvent être exemplaires en invitant à observer notre objet non pas au filtre de l'altérité, mais plutôt à celui d'un commun exacerbé.

\section{Réactions des parents : ce dont témoignent les interviewés}

7 On peut classer les réactions parentales telles qu'elles ont été rapportées par les interviewés dans les rubriques présentées dans le tableau ci-dessous.

8 La lecture du tableau doit être nuancée car certaines catégories peuvent avoir des significations différentes, voire contradictoires, ou encore évoluer, par exemple si l'élève a connu plusieurs conseils de discipline. L'aide à la réinscription n'implique pas systématiquement de soutien moral. Il s'agit simplement pour le parent de trouver un nouveau point de chute pour l'enfant afin d'éviter la déscolarisation ou de satisfaire à l'obligation scolaire.

9 Le recours aux tiers prend également des formes variées : il peut s'agir d'un membre de la famille proche ou par alliance (oncle, marraine) ou d'un spécialiste (psychologue, éducateur spécialisé ou avocat) ${ }^{8}$. Dans près de la moitié des cas, le recours aux spécialistes est lié à des poursuites judiciaires accompagnant l'exclusion de l'établissement (8 cas parmi les 19 renseignés).

10 Au-delà de ces nuances, les discours des interviewés témoignent de la cristallisation des tensions au sein de la famille, d'une distance au sein du domicile parental ou même d'un éloignement physique de l'élève. C'est aux motifs et aux conséquences de cet éloignement du domicile parental que sera consacré l'essentiel des développements à suivre, tant les répercussions des conseils de discipline au sein des familles et sur l'avenir des jeunes semblent n'avoir jamais attiré l'attention en France. 
Tableau 1

Réactions parentales après le conseil de discipline

\begin{tabular}{|l|l|}
\hline $\begin{array}{l}\text { Perception par l'adolescent des réactions parentales } \\
\text { après le CDD }\end{array}$ & $\begin{array}{l}\text { Occurrence } \\
\text { dans } \\
\text { corpus }\end{array}$ \\
\hline Soutien actif (moral et/ou démarches pour une nouvelle inscription)
\end{tabular}

11 Notes: Ce tableau a été élaboré à partir d'une lecture systématique du corpus à l'aide d'une grille d'analyse focalisée

sur les parents et leurs attitudes après le CDD et l'exclusion.

Table 1

Parental reactions after disciplinary board

Dans la majeure partie des cas, les récits recueillis auprès des exclus témoignent du fait que l'exclusion provoque de la déception chez les parents, plus rarement de l'incrédulité ou de l'incompréhension. La majorité des personnes rencontrées témoigne d'un parcours déviant relativement marqué dans l'établissement, le CDD consacrant au niveau de l'établissement une carrière scolaire émaillée d'incidents jugés menaçants pour l'ordre scolaire ${ }^{10}$.

« Ma mère a tellement de tableaux d'honneur à moi dans le primaire. Elle a ça dans une pochette cartonnée, et puis elle me dit des fois : "Regarde le délinquant que tu as fait, putain !”.» [Michel-André, 29 ans, exclu en $5^{\mathrm{e}}$ puis en $3^{\mathrm{e}}$.]

13 Les personnes interrogées évoquent l'embarras parental qui débute avant même la réunion de l'instance disciplinaire. La convocation des (ou du) parent(s) dans le bureau 
du chef d'établissement, la lassitude, la certitude que les "jeux sont faits", la perspective d'être exposés aux critiques de la communauté scolaire dissuadent certains d'entre eux de se rendre au CDD. Étrangers au système scolaire, les parents de milieu populaire dénoncent un sentiment d'impuissance face aux professionnels de l'éducation (Blair, 2001). Cette défection s'inscrit dans la logique des stratégies d'évitement mises en place par les parents d'origine populaire pour se protéger d'une imposition des normes dominantes par l'institution scolaire (Lorcerie \& Cavallo, 2002).

La mère de Jean-Louis ira chercher la notification d'exclusion au collège le lendemain du CDD. L'élève affronte seul l'instance. Le père, chômeur, n'est jamais intervenu dans l'éducation de l'enfant et ne dira pas un mot concernant l'exclusion. «Bon, mon père vivait avec nous, mais je préfère dire que j'ai été élevé par ma mère, parce que c'est elle qui m'a élevé en fait. Ma mère, et mes frères et sœurs ". Concernant l'absence de la mère au CDD : «Ça s'est passé le soir. Pas trop tard non plus. Il devait être 6 heures. Bon, comme c'était la période... Il faisait quand même assez noir. Quand elle sort du travail, elle est fatiguée, donc elle n'avait pas envie de reprendre la route pour aller là, pour entendre qu'on m'a exclu. » [Jean-Louis, 26 ans, exclu de $4^{\mathrm{e}}$, CAP vente, projette de créer une entreprise.]

À l'humiliation des parents, aux pleurs des mères qui assistent souvent seules aux conseils de discipline, succèdent dans quelques cas des formes de violence verbale ou physique dans l'intimité du foyer.

«- Quand ta maman a su que tu allais être exclu, comment elle a réagi?

- Ah ! Elle était énervée au début, elle a dit ça à mon papa [dont elle est séparée]. Elle a voulu me taper, mais j'ai couru. Et puis elle m'a rien fait. Elle était énervée contre moi, elle a dit ça à mon papa. Il m'a appelé pour me dire des remarques. C'est tout.

- Elle est restée longtemps sans te parler?

- Pfff... peut-être deux mois, ouais, deux mois. Je la voyais, je lui disais bonjour, elle ne me donnait pas d'argent. Elle me disait jamais rien. Elle me disait : "Mwen pani lagen" ${ }^{\prime 1}$. Y'avait rien. " [Pedro, 18 ans, exclu en $4^{\mathrm{e}}$ et en seconde. Prépare un BEP dans l'agroalimentaire.]

Céline [13 ans, scolarisée en $5^{\mathrm{e}}$ lors de l'entretien] s'est fait exclure de $6^{\mathrm{e}}$ suite à des tensions avec plusieurs enseignants et à la falsification de son carnet de correspondance. Pendant le conseil de discipline, elle n'osera rien dire. «J'avais peur de la réaction de ma maman envers moi. " Les parents ont «mal réagi, très mal: ils ont crié après moi et ils m'ont donné des coups. Ils n'ont pas pris [pas accepté] ça. » Ce type de châtiments corporels s'inscrit dans ce que Thin appelle l'exercice d'une "contrainte extérieure », à savoir l'« interruption immédiate des actes répréhensibles et leur sanction dès que ceux-ci sont repérés. [...] Le travail parental consiste davantage à surveiller, à interdire ou à limiter les actions des enfants qu'à inculquer ou à discuter des règles de sécurité ou de moralité auxquelles ces derniers soumettraient leurs comportements " (Thin, 2009, p. 73)

15 À la violence s'ajoute parfois ce qui peut être décrit comme une attitude d'abandon ou de rejet de la part des parents qui semblent désormais refuser d'assumer une responsabilité vis-à-vis de leurs enfants.

« [Après le deuxième $\mathrm{CDD}$ ] Ma maman, elle m'a dit de régler mes trucs moi-même ce coup-ci, parce qu'elle a fait le maximum pour moi et j'ai refait la même chose. Donc elle a dit comme quoi elle refait plus rien pour moi, que je vais me démerder, que je vais me débrouiller pour faire mes démarches, pour trouver un truc à faire. » C'est une cousine de quatre ans son aînée qui l'aidera à trouver un nouvel établissement, mais quand le comportement d'Yvan se dégrade à nouveau, l'établissement refusera de rencontrer cette interlocutrice et exigera de voir les parents. [Yvan, 20 ans, deux fois exclu en seconde. En apprentissage, plomberie.] 
Cette attitude d'abandon par la cellule familiale, décrite par plusieurs interviewés, suggère que les parents semblent ne plus croire en l'efficacité de la persuasion ou même du redressement par châtiments corporels pour corriger le comportement de leurs enfants ${ }^{13}$. Dès lors, les difficultés de l'enfant sont naturalisées, et il s'agit de composer avec. Neuf interviewés décrivent des parents qui, littéralement, leur «tournent le dos » après le verdict du CDD. Dans douze cas, les interviewés décrivent l'apparition d'une distance, voire d'une tension durable avec les parents.

Pour David l'exclusion de l'établissement est vécue comme un élément qui va l'isoler encore davantage à l'intérieur du cercle familial. Alors que sa mère et son beau-père insistent pour qu'il aille dans un établissement de bonne réputation où ses sœurs aînées ont déjà réussi, lui ne s'y sent pas à sa place et développe un comportement perturbateur. David vole, rackette, arnaque, se prend "une volée " pour avoir dérobé de l'argent à sa mère. Le conseil de discipline vient donner un tour plus «public » et officiel à cette carrière déviante. "Ma mère, depuis ce jourlà, elle me voyait un peu comme le vilain petit canard quoi. [...] J'étais devenu l'insupportable, le siré $!^{14}$ " David semble s'affirmer contre le modèle de réussite incarné par la réussite des aînées, il rejette par ailleurs son beau-père. De plus en plus isolé au niveau familial, il pourra poursuivre sa scolarité en entrant dans un établissement populaire et au milieu d'une classe difficile. Cet environnement dans lequel il se sent plus à l'aise le voit poursuivre jusqu'à un baccalauréat technologique. [David, 29 ans, exclu de $5^{\mathrm{e}}$ ]

Cette attitude parentale peut être interprétée comme du fatalisme - l'enfant est « comme cela ", on ne le changera plus - mais aussi comme une stratégie de retrait vis-àvis du monde scolaire (Périer, 2005). Il s'agit cette fois de se dérober à l'influence des jugements scolaires au sein même de la famille pour se protéger soi-même ainsi que le reste de la fratrie ${ }^{15}$. Ce retrait ne concerne plus l'établissement scolaire avec lequel on chercherait à limiter les interactions, il s'exerce désormais vis-à-vis de l'élève par lequel les jugements scolaires défavorables sont importés au sein même du milieu familial.

Dans plusieurs cas, la tension que cristallise le CDD au sein des familles n'est jamais complètement résolue ou continue de marquer l'élève. Pour les parents de Liam, cette confiance ne commence à se restaurer qu'une fois obtenu le premier diplôme: "Ils m'ont mis une certaine pression en me disant que si je n'avais pas le diplôme, il faudrait que je trouve quelque chose à faire, que je n'allais pas rester à la maison à ne rien faire. [...] Ils n'y croyaient pas, ils avaient baissé les bras. Ils n'y croyaient pas. » La motivation de Philippe viendra de la volonté de "changer le regard que mes parents avaient envers moi. » De fait, la défiance parentale peut conduire à deux types de résolution par l'enfant : une prise de conscience ou une rupture avec la famille.

\section{Les tiers : entre dernier recours et double rupture}

Les sorties de crises impliquent bien souvent le recours à des ressources extérieures à la famille ou le placement chez des tiers. Dans les deux cas, il s'agit de recourir aux ressources scolaires et culturelles familiales disponibles auprès de tiers permettant de prendre appui sur des personnes considérées par les parents comme "plus aptes » à aider au maintien de l'élève dans sa scolarité.

Dans le cas d'Yvan [20 ans, exclu deux fois de seconde, en apprentissage], c'est une cousine qui le prend en charge après sa deuxième exclusion de seconde. C'est elle qui va devenir l'interlocutrice du nouvel établissement pour la réinscription de l'élève. La 
mère semble avoir abandonné tout espoir de corriger le comportement de son fils, Yvan appartient à une fratrie très turbulente de trois enfants qu'elle élève seule.

21 Cette aide peut porter sur les seules démarches administratives en recourant à un membre de la famille, car on estime qu'il est plus «légitime» (professionnels de l'Éducation nationale, diplômés du supérieur). Elle peut être également matérielle (hébergement) et morale (encadrement moral de l'élève). Ce tiers est une ressource pour la famille, car il peut instaurer un suivi scolaire déconflictualisé (Lorcerie \& Cavallo, 2002).

Bien intégré dans la cité auprès des "gars», Michel-André voulait prouver aux autres sa valeur. Plutôt que de braquer ou racketter d'autres élèves, il décide de " braquer l'établissement ». Il dérobe en bande le matériel informatique, les sonos, etc. « On m'a envoyé dans une sorte de petit centre, et il y avait plein de petits Jésus sur le mur. [...] Mais je ne suis pas resté longtemps, parce que ma marraine connaissait des gens. Des avocats. Le juge même... il connaissait ma marraine.» Nouveau départ dans un quartier de la banlieue de Fort-de-France où il va vivre avec sa marraine. "Elle a vu un oncle à moi, qui est principal adjoint. On lui a expliqué le cas, et puis bon comme c'était mon oncle... » Michel-André intègre donc un nouvel établissement. En $3^{\text {e }}$, on le renvoie une seconde fois, car les avertissements se sont accumulés. Cette fois, c'est une tante qui l'héberge dans une commune du centre de l'île. L'élève se déscolarise au bout de quelques mois. En intégrant une nouvelle école dans une commune plus éloignée du centre urbain de l'̂̂le, Michel-André parvient à obtenir un BEP sanitaire et social. [Michel-André, 29 ans, exclu en $5^{\mathrm{e}}$ puis en $3^{\mathrm{e}}$.]

On voit ici les parents s'appuyer sur la solidarité familiale. Dans la tentative de rescolarisation de Michel-André interviennent une marraine, un oncle et une tante. Son oncle autorise son inscription dans l'établissement dont il est principal adjoint, les deux femmes acceptent de l'héberger pour le rapprocher de son nouvel établissement. Le recours à des tiers, notamment à des membres de la famille, est une pratique fréquente puisqu'ils sont mentionnés par la moitié des informateurs.

Dans le cas ci-dessous, les parents posent un ultimatum à leur fille, celle-ci refuse de céder. L'hébergement par la grand-mère devient un compromis.

Le père de Michelle est agriculteur, la mère élève les quatre enfants. Michelle est la seule de sa fratrie à connaître des difficultés scolaires et comportementales régulières. Les parents la changent d'environnement, font appel à des spécialistes, mais rien n'y fera. Michelle est passée experte dans la dissimulation, ils la croient assidue dans l'établissement privé qui grève leur budget (4 000 euros/an). Quand les parents sont convoqués au conseil de discipline, ils tombent littéralement des nues. Ils sont « déçus » et « dégoûtés ». « Mon pater a refusé d'aller à la convocation, il a dit qu'il avait fini avec moi et que si je voulais gâcher ma vie, c'était mon problème. » La mère ne se rend pas non plus à la convocation. À l'issue du conseil de discipline, Michelle se voit poser un ultimatum : «Mes parents ont décidé que je devais choisir, soit je restais à la maison et je me conformais aux règles qu'ils avaient fixées et je trouvais quelque chose à faire de ma vie, ou bien je prenais la porte et j'allais vendre mon herbe dans la rue et traîner avec mes amis. » Michelle va s'installer chez sa grand-mère, ce qu'elle décrit comme une libération. [Michelle, 21 ans, exclue de $1^{\text {re }}$, BEP électrotechnique, en contrat à durée déterminée.]

Pour les couples séparés, la garde étant généralement assurée par l'un des deux parents, une solution peut être d'envoyer l'enfant vivre chez l'autre parent et de solliciter une rescolarisation dans un établissement proche du nouveau domicile.

La scolarité de Mickaël est jalonnée par des allers-retours Métropole - Martinique.

Quand il se fait exclure en $6^{\mathrm{e}}$, il va vivre chez sa mère en métropole. Quand, après un 
redoublement, il est à nouveau exclu en fin de $5^{\mathrm{e}}$, il revient vivre chez son père en Martinique, "pour se faire serrer les vis » et parce que sa mère "en a ras la casquette ». Mickaël est soutenu par des parents qui investissent beaucoup d'énergie pour le maintenir scolarisé, l'aider à conserver un bon niveau scolaire et lui offrir un environnement de travail favorable. [Mickaël, 16 ans, exclu de $6^{\mathrm{e}}$ et $5^{\mathrm{e}}$ actuellement déscolarisé, aucun diplôme.] 1'écartant du cadre qui était celui de ses interactions scolaires, pour en adopter un nouveau. Pour les membres de 1'institution, il s'agit de provoquer une rupture avec les pairs dont l'influence est parfois néfaste, permettre de fréquenter de nouveaux enseignants et personnels de direction avec lesquels - «normalement »- les relations ne sont pas teintées d'un passif. C'est ce «nouveau départ» qui peut justifier l'exclusion ${ }^{16}$.

\section{Une double exclusion}

Ce qui frappe pourtant à la lecture des entretiens, c'est pour nombre de ces élèves le caractère double de cette «mort sociale » définie par Goffman (1952/1989), et dont l'impact est sans doute moins perçu par les professionnels. Mourir socialement, c'est être privé de l'un de ses rôles sociaux, c'est voir disparaître ou compromise une de nos facultés sociales. Et Goffman de s'interroger sur les «manières de gérer le passage d'un rôle que nous jouons habituellement à 1'état où nous nous trouvons lorsque nous ne voulons plus le jouer » (Goffman, 1952/1989, p. 298). L'épreuve qui touche durement les expulsés, les licenciés, les retraités, les excommuniés, les victimes de faillites économiques ne s'applique pas moins aux élèves exclus. Le CDD et l'éventuelle exclusion définitive de l'établissement ne consacrent pas seulement le déracinement scolaire de l'élève, ils viennent également menacer l'enfant au sein de son environnement familial.

Sidney est en $4^{\mathrm{e}}$, il peine à intégrer l'ordre scolaire (refus de travail, mauvais comportement, choisit ses cours en fonction des enseignants qu'il apprécie ou pas, insulte une surveillante) jusqu'au jour où une bagarre dégénère et où il frappe un 
élève avec une paire de ciseaux. «Mon papa, il ne comprenait pas comment j'ai pu faire ça. Durant quinze jours, il était vraiment bizarre, il ne parlait pas. Il était dans un sale état. Il était, je ne sais pas, dans l'incompréhensibilité. Ma maman non plus ne comprenait pas, mais rna maman elle est plus psychologue, elle essayait de comprendre. Il ne comprenait pas, il était vraiment perdu.

Et après le conseil, ils t'ont soutenu?

Ils ne m'ont pas soutenu. Ah non, pas du tout. Mon papa déjà rn 'a dit de rn e mettre bien parce que c'est la dernière fois que ça m'arrivait. Et ils n'ont puni. Mais ils ne m'ont pas aidé genre moralement. Ma maman a essayé de le faire, elle était fâchée, mais elle a essayé de remonter avec moi. Mon papa, c'était: “"Tant pis pour moi”. [...] Mon papa après un mois et plus il est resté très bizarre. Même très bizarre. » Les parents de Sidney vont faire appel à un psychologue qui va voir l'enfant une demidouzaine de fois.

L'année suivante, le père reçoit une lettre le convoquant pour régler les problèmes d'absentéisme de Sidney. La mère est en France métropolitaine. «Il m'a demandé : “Tu as été où ? Tu fais quoi ?" Il a mis la feuille sur la table. Il a demandé à rna sœur de s'occuper de ça. Et là aussi, il est resté bouche bée. » [Sidney, 17 ans, scolarisé en lycée général.]

Pour certains élèves, la coupure avec 1'environnement scolaire se double donc d'une fracture familiale qui débute avec la déception puis l'amertume des parents, celle-ci prenant la forme de violences physiques et morales, d'un ostracisme à 1'intérieur de la famille ou d'une mise à 1'écart physique de la cellule familiale. L'exclusion d'un établissement scolaire n'est pas sans effet sur 1'intégration familiale du jeune qui peut connaître une « double exclusion », à la fois scolaire et familiale.

\section{Le « filet de protection » des soutiens relationnels}

Dans deux cas, Éric et Jimmy, les ressources familiales peuvent être maladroitement mobilisées et s'avérer contre-productives. Dans le cas d'Éric [30 ans, exclu de terminale, baccalauréat professionnel], le père lui-même enseignant vient rencontrer l'équipe pédagogique de son fils, mais c'est en état d'ivresse qu'il se présente à1'établissement; dans celui de Jimmy [27 ans, exclu de $4{ }^{\mathrm{e}}$, déscolarisé], le père toxicomane vient menacer un personnel de direction avec plusieurs autres «zombies» (drogués). Dans le second cas, cette mobilisation maladroite vaudra à l'élève plusieurs jours d'exclusion temporaire de l'établissement.

31 À l'inverse, les soutiens familiaux peuvent s'avérer essentiels à la poursuite de la scolarité des élèves. L'épreuve peut resserrer les liens autour du jeune. Anthony, 23 ans, est menacé d'exclusion en première professionnelle après qu'un sachet de drogue soit tombé de sa poche lors d'un cours. Anthony, qui est en alternance, voit se mobiliser autour de lui son employeur, ses parents, sa petite amie, ses enseignants et un cousin qui lui aussi avait connu des problèmes dans le même établissement, mais qui « s'en est sorti ». Tous attestent auprès de l'institution du sérieux du garçon, plaident une erreur de jeunesse et demandent une deuxième chance. Anthony sera finalement autorisé à poursuivre sa scolarité et obtiendra son baccalauréat professionnel.

En France, comme dans la plupart des pays industrialisés, les besoins en main-d'œuvre non qualifiée et les réseaux familiaux n'assurent plus, comme par le passé (Willis, 1978), un accès privilégié au monde du travail ${ }^{17}$. Ces formes d'insertion professionnelle et de solidarité anciennes ne sont pas remplacées par les liens noués par les jeunes dans « la rue ». L'exclusion scolaire opère dès lors comme un révélateur de la fragilité sociale de l'élève soulignant a posteriori le caractère sécurisant de l'établissement scolaire et de 
l'intégration familiale. Or, de par leur fonction symbolique, la puissance de leur ritualisation, les CCD ébranlent les « supports » relationnels des jeunes (Castel, 1991 ; Castel \& Haroche, 2001) déjà fragilisés par leur trajectoire scolaire.

«Le terme de "support" peut avoir plusieurs acceptions, mais je le prends ici au sens de condition objective de possibilité. Parler de support en ce sens, c'est parler de "ressources", ou de "capitaux" au sens de Bourdieu ; c'est la capacité de disposer de réserves qui peuvent être de type relationnel, culturel, économique, etc., et qui sont les assises sur lesquelles peut s'appuyer la possibilité de développer des stratégies individuelles. [...] Pour entrer en interrelation avec les autres, l'individu doit avoir ces conditions sociales pour être déjà un individu, disposer de ressources pour le faire. [...]. Ce qui m'intéresse, c'est la question des conditions de possibilité nécessaires pour être un individu, ou une personne, ou un acteur, ou un sujet. » (Castel \& Haroche, 2001, pp. 32-33.)

Le rituel institutionnel et les règles administratives de l'exclusion - le CDD et la réinscription automatique des élèves encore soumis à l'assiduité scolaire - masquent la fragilisation des soutiens familiaux de 1'individu et les conséquences pour son devenir. L'apparente continuité scolaire camoufle la rupture qui s'opère au sein des familles. Les données témoignent de ce processus: sur l'ensemble de nos participants, dix jeunes ont quitté le domicile familial après avoir été exclus de leur établissement scolaire. Parmi eux, six étaient sous l'autorité parentale de leur seule mère.

Les trajectoires scolaires d'Alain et d'Alice illustrent l'importance de cette " protection rapprochée » (Castel, 1991) que représentent ces « supports » familiaux.

Alain a redoublé la $6^{\mathrm{e}}$ suite à la séparation de ses parents. Sa mère était commerçante, son père fonctionnaire. Alain vit avec sa mère, il n'a pas été un élève très travailleur.

Alain n'exprime aucun regret vis-à-vis de son exclusion, car il pense que cette expérience lui a permis de rompre avec des comportements inadmissibles de la part de ses enseignants. « On ne m'aimait pas et je ne les aimais pas non plus. » L'origine du conflit serait des mots déplacés de l'enseignant d'histoire-géographie sur la profession de la mère de l'élève : « Le jour où il a appris que ma mère exerçait une profession libérale, pour lui ça voulait dire que j'étais un nanti, extrêmement riche. En fait, il avait tous ces clichés en tête. [... ] Il ne mâchait pas ses mots. " Suite à son exclusion, Alain intègre un établissement professionnel et prépare « un truc court » (BEP). Il est reçu par son nouveau proviseur qui lui demande : «Qu'est-ce que vous faites là ? » «Comment? Je viens m'inscrire. »À la vue de ses résultats scolaires, le proviseur répond : «Votre place n'est pas ici, votre place est au lycée [général]. » $\mathrm{Au}$ lycée professionnel, ses notes ne sont jamais inférieures à 16/20. Alain ne poursuit pas ses études au-delà de son BTS en action commerciale, car il doit soulager ses parents en reprenant le commerce de sa mère. "Je n'en avais pas l'intention, je voulais faire une tout autre profession, ça ne m'intéressait pas forcément. J'ai décidé de reprendre le flambeau quand j'ai compris qu'il fallait être reconnaissant dans la vie. »

Sa mère l'a soutenu, mais elle ne prendra conscience que tardivement de la mauvaise foi de l'enseignant qui a poussé à son exclusion : «Elle m'en a parlé et elle s'en voulait de ne pas avoir suffisamment prêté l'oreille et d'avoir trop fait confiance au système, aux profs, au cadre scolaire quoi. Il était trop tard, mais bon, je pense que je n'ai pas mal tourné par rapport à cette injustice que j'ai vécue pendant de longues années. » [Alain, 41 ans, exclu de seconde gestion, il avait 17 ans, actuellement commerçant.]

L'expérience d'une discrimination sociale va impacter le devenir de l'élève.

« Les individus sans protection sont aussi des individus, et ils vi vent cette situation comme des individus, ne serait-ce qu'à travers la souffrance d'être dans cet état. Mais d'un autre point de vue, on pourrait dire qu'ils sont moins des individus au 
sens de pouvoir développer des stratégies personnelles, d'avoir par eux-mêmes et pour eux-mêmes des marges de manœuvre. » (Castel \& Haroche, 2001, pp. 121-122.) Alain va « s'en sortir » en reprenant 1'affaire familiale alors qu'il avait d'autres aspirations. À cet impact sur son devenir s'ajoute celui de 1'incompréhension durable qui s'était installée entre lui et sa maman. L'expérience d'Alice est différente, les soutiens relationnels sont mobilisés très tôt et durablement. L'argent et l'influence des membres de cette famille issue de la bourgeoisie de province lui permettent d'échapper à des ennuis judiciaires et d'opérer très tardivement un retour réussi sur la scène scolaire.

Alice a six ans quand elle est confiée à ses grands-parents. Sa mère ne semble pas en mesure de faire face à ses responsabilités vis-à-vis d'elle.

Ses grands-parents, représentants de la bourgeoisie industrielle, la placent en internat dès le primaire. Elle sera interne presque toute sa scolarité, quasi exclusivement dans des établissements privés. Alice se présente comme ayant été une élève particulièrement pénible et peu travailleuse, cumulant avec les années de grosses lacunes. En 4 e, elle «tabasse » une camarade : «Donc ses parents ont porté plainte. Finalement les parents ont retiré leur plainte, et on a pu étouffer tout ça. Je ne sais pas trop comment... » La même année, elle insulte son enseignante d'anglais, ce qui provoque sa première exclusion.

Après avoir redoublé la $4 \mathrm{e}$ dans un autre collège, elle se fait exclure cette fois pour avoir fumé et apporté de l'alcool dans l'établissement. Une prise de conscience se fait en deux temps : dans le bureau de la principale d'un lycée où sa famille cherche à l'inscrire, mais qui la refuse : «Ils m'ont dit pourquoi ils ne m'ont pas prise. Et là je me suis dit: “O.K., voilà les conséquences de tout ce que j'ai fait avant." Et là, j'ai vraiment eu peur parce qu'aucune école ne voulait de moi, aucune... aucune... aucune... Pourtant, ma grand-mère avait beaucoup de contacts. En plus, elle a une force de persuasion qui est assez impressionnante. Et je me suis dit: "Même avec ça, on ne veut pas me prendre." Et là, je me suis dit: "Je crois que j'ai atteint la limite."” Après une première littéraire qui l'ennuie "à mourir ", elle se réoriente en BEP : "C'est un petit peu quand je suis arrivée à mon BEP, je crois qu'au bout de deux mois j'ai entendu parler du métier d'éducateur spécialisé, ça m'a fait un "tilt" quoi. Je me souviens, je me suis dit: “C'est ça que je veux devenir". » Alice obtient son $\mathrm{BEP}$, un baccalauréat professionnel, puis décroche son diplôme d'éducateur spécialisé avec de bons résultats.

Interrogée sur la façon dont elle analyse sa situation à l'époque, sur ce que les acteurs $\mathrm{du}$ monde scolaire auraient pu mettre en œuvre pour favoriser son raccrochage scolaire, Alice répond :

«Ben justement, c'est une manière d'y penser qui vient $d u$ fait que j'ai $f$ ait ma formation d'éduc' spé, je me dis qu'il y avait forcément quelque chose à faire. Maintenant, je ne pense pas. Enfin de manière objective, il fallait que je passe par ça. [... ]Même s'ils avaient mis des choses en place, je ne pense pas que ça aurait réussi.

- Finalement il y aurait des moments où ça n'est pas la peine de forcer les jeunes à se raccrocher?

- Ouais. [Réfléchit] Après, je pense ça, mais d'un autre côté, si on les laisse se planter, il faut être sûr qu'il y a les personnes autour qui pourront les rattraper. Parce que moi, mes grands-parents ont toujours été là, et il y a aussi le fait que comment dire ? ils ont les moyens aussi financiers et autres qui fait que j'ai pu m'en sortir. Mais quand on vient d'une famille où on a rien, là je pense que c'est difficile de laisser son enfant aller dans ce sens-là. Là, à mon avis, ça ne fera pas les mêmes résultats. "[Alice, 26 ans, exclue de $4^{\mathrm{e}}$ et de $3^{\mathrm{e}}$, diplôme d'État d'éducateur spécialisé.] 


\section{Discussion}

37 Parmi les ressources mobilisables à1'occasion de 1'exclusion d'un établissement scolaire, les relations familiales sont cardinales (Wright et al., 2005). Pour certains jeunes, notamment les plus démunis (foyers monoparentaux, faibles revenus, faible capital social), 1'exclusion scolaire provoque ou accélère l'effritement du "support » familial. En effet, même si l'institution ne fait que consacrer négativement une trajectoire scolaire marquée par des comportements non scolaires, l'exclusion définitive d'un établissement scolaire ébranle ce qui apparaît comme le dernier soutien sur lequel les jeunes peuvent essayer de bâtir leur devenir, à savoir la solidarité familiale.

38 Ces premiers résultats suggèrent l'importance de préparer et d'accompagner les familles dans le traitement de l'exclusion scolaire. Toute la difficulté pour les acteurs amenés à s'exprimer en pareils cas (professionnels, membres élus aux instances disciplinaires) réside dans l'appréciation des ressources familiales mobilisables autour du jeune et 1'anticipation des réactions parentales. Cela apparaît d'autant plus crucial que les motifs des réactions parentales sont parfois difficiles à distinguer. Par exemple, la redomiciliation de l'élève peut tout autant prendre la forme d'une volonté de bannir l'enfant de la cellule familiale que de celle, pragmatique, de le rapprocher du nouvel établissement scolaire dans une région où les transports publics posent problème. Pour une fraction d'élèves, ceux dont les soutiens relationnels sont les plus précaires, il apparaît que 1'exclusion de 1'établissement scolaire n'est définitivement pas une " seconde chance ». Elle fonctionne au contraire à la manière d'une double peine. Chaque année, en mettant à l'épreuve les «supports » familiaux d'environ 17000 élèves, le système éducatif français participe du processus de "désaffiliation sociale » (Castel, 1991) d'une fraction de cette jeunesse. La vulnérabilité des plus précaires est exacerbée quand l'expérience de l'exclusion consacre négativement une carrière scolaire (provoquant une fragilisation de l'affiliation scolaire) et ébranle parfois durablement les solidarités familiales (dont les conséquences sont la fragilité relationnelle) dans un contexte où le marché de l'emploi n'est pas favorable aux peu diplômés (vulnérabilité professionnelle).

Dès lors, l'exclusion définitive d'un établissement scolaire ne peut plus être conçue dans le seul cadre scolaire tant cette problématique rejoint celle de la citoyenneté et de 1'exclusion sociale : «De façon cruciale, 1'exclusion sociale soulève le problème de la possibilité pour les personnes de faire des choix authentiques, des 'engager dans des processus de prise de décision concernant leur propre existence, de contribuer à la qualité de vie de leur communauté et de peser dans les décisions collectives » (Blyth \& Milner, 1993, p. 266). 


\section{BIBLIOGRAPHIE}

Beaud, S. (2002). 80 \%, au bac... et après ? Les enfants de la démocratisation scolaire. Paris : La Découverte.

Becker, H S. (1985). Outsiders. Études de sociologie de la déviance. Paris : Métailié.

Berthet, T, \& Zaffran, J (éd.). (2014). Le Décrochage scolaire. Acteurs et enjeux de la déscolarisation. Rennes : Presses Universitaires.

Blair, M. (2001). Why Pick on Me? School Exclusion and Black Youth. Stoke-on-Trent/Sterling: Trentham Books.

Blyth, E., \& Milner, J. (1993). Exclusion from school: a first step in exclusion from society? Children \& Society, $7(.3), 255-268$.

Castel, R (1991). De l'indigence à l'exclusion, la désaffiliation. Précarité du travail et vulnérabilité relationnelle. In J Donzelot (éd.), Face à l'exclusion. Le modèle français. (pp. 137-168). Paris : Éditions Esprit

Castel, R, \& Haroche, C. (2001). Propriété privée, propriété sociale, propriété de soi. Paris : Fayard. Chevit, B. (2003). Commission disciplinaire et conseil de discipline. Déviance et Société, 27(4), 483-503.

Cullingford, C. (1999). The causes of exclusion. Home, school and the development of young criminals. London/Sterling: Kogan / Page.

Esterle-Hedibel, M. (2006). Absentéisme, déscolarisation, décrochage scolaire, les apports des recherches récentes. Déviance et Société, 30(1). 41-65.

Fotinos, G., \& Horenstein, J. M. (2011). La qualité de vie au travail dans les lycées et collèges. Paris : MGEN.

Geay, B., Oria, N., \& Fromard, L. (2009). La remise en ordre symbolique de l'institution. Les conseils de discipline dans l'enseignement secondaire. Actes de la recherche en sciences sociales, 178, 62-79.

Goffman, E. (1952/1989). Calmer le jobard : quelques aspects de l'adaptation à l'échec. In I. Joseph, R Castel, \& J. Cosnier (éd.), Le parler frais d'Erving Goffman. Paris : Éditions de Minuit

Hayden, C. (1997). Children excluded from primary school. Buckingham/Philadelphia: Open University Press.

Hayden, C. (2001). Facteurs de risque et exclusion de l'école. In E. Debarbieux, \& C. Blaya (éd.), Violence à l'école et politiques publiques (pp. 95-115). Paris : ESF

Hayden, C. (2003). Responding to exclusion from school in England, Journal of Educational Administration, 41(6), 626-639.

Lorcerie, F, \& Cavallo, D. (2002). Les relations entre familles populaires et école. Les Cahiers Millénaires, 24(3), 5-24

MEN (2012). Repères et références statistiques sur les enseignements la formation et la recherche. Paris : Ministère de l'Éducation nationale.

MEN (2014). Géographie de l'École. Paris : Ministère de l'Éducation nationale. 
Millet, M, \& Thin, D. (2005). Ruptures scolaires. L'école à l'épreuve de la question sociale. Paris : Presses Universitaires de France.

Munn, P, \& Lloyd, G. (2005). Exclusion and Excluded Pupils. British Educational Research Journal, 31(2), 205-221.

Périer, P (2005). École et familles populaires : Sociologie d'un différend. Rennes : Presses Universitaires.

Pomeroy, E. (2000). Experiencing exclusion. Stoke-on-Trent: Trentham Books.

Renahy, N. (2005 / 2010). Les gars du coin. Enquête sur une jeunesse rurale. Paris : La Découverte.

Thin, D. (2009). Un travail parental sous tension : les pratiques des familles populaires à l'épreuve des logiques scolaires. Informations sociales, 154(4), 70-76.

Tondellier, M (2006). Un établissement scolaire sous pression. Analyser les conseils de discipline au collège Balzac. Déviance et Société, 30(2), 179-202.

Valentin-Marie, C., Temporal, F., Condon, S., Chanteur, B., Macheclerc, M, \& Breton, D. (2012). Migrations, famille et vieillissement défis et enjeux pour la Martinique. Antiane Échos, 20, 1-8.

Willis, P (1978). L'école des ouvriers. Actes de la recherche en sciences sociales, 24(1), 50-61.

Wright, C., Standen, P, John, G., German, G., \& Pate!, T (2005). School Exclusion and Transition In to Adulthood in African Caribbean Communities. York: Joseph Rowntree Foundation.

\section{NOTES}

1. Pour une présentation synthétique de ces travaux, cf. Esterle-Hedibel (2006), pour des apports plus récents, cf. Berthet et Zaffran (2014).

2. En Angleterre, les exclusions définitives concernant quatre fois sur cinq des garçons, majoritairement les enfants de milieux défavorisés, de familles monoparentales ou recomposées et les élèves rencontrant des «besoins éducatifs spéciaux • (special education needs). La dimension ethnique joue également puisque les enfants issus de familles afro-caribéennes sont également surreprésentés (Hayden, 2001, 2003).

3. La recherche anglo-saxonne a développé un intérêt précoce sur l'exclusion (Hayden, 1997) et plus prononcé pour l'analyse de l'expérience des élèves exclus replacée dans les contextes scolaires et/ou familiaux (Cullingford, 1999 ; Pomeroy, 2000 ; Blair, 2001; Munn \& Lloyd, 2005).

4. Pour une analyse fine des trajectoires de rupture scolaire, se reporter à l'ouvrage de Millet et Thin (2005).

5. De façon à contourner les réticences (des chefs d'établissement, des parents) à aborder ce sujet en milieu scolaire, nous avons recouru aux réseaux relationnels des étudiants des promotions 2009 à 2013 de licence 3 de sciences de l'éducation de l'VAG. Cette analyse doit beaucoup à l'investissement de ces étudiants (C. Cellamen, J. Lange, M.-C. Radom, S. On no) que je tiens à remercier. Les modalités de constitution de l'échantillon étant largement contingentes, les informateurs peuvent avoir été interrogés «à chaud» ou bien de nombreuses années après les événements. Cette diversité des discours recueillis n'est pas sans poser question quant au caractère plus ou moins réflexif des discours, mais il s'est agi là de concessions pour accéder à un terrain rétif à l'enquête.

6. Quant au fonctionnement du système éducatif, après une période de « rattrapage » au niveau structurel et quantitatif (taux de scolarisation, taux de réussite aux diplômes, etc.), son organisation est la même qu'en France métropolitaine depuis la départementalisation de 1946.

7. Familles populaires, monoparentales, exposition à la pauvreté, les points de comparaison ne manquent pas avec le cas anglais (Pommeroy, 2000). 
8. Si l'on distingue deux populations, celle dont l'exclusion implique un signalement judiciaire et celle dont les conséquences administratives ne dépassent pas le cadre scolaire, le recours au psychologue est un pivot. Pour la seconde population, les tiers mobilisés appartiennent exclusivement à la famille ; pour la première, éducateurs spécialisés et avocats interviennent. Si le psychologue est mobilisé par certaines familles de ces deux populations, c'est dans les familles les plus fragiles - milieux populaires, familles monoparentales - que son intervention paraît la moins comprise et, peut-être, la moins efficace.

9. Plusieurs éléments peuvent apparaître dans un même discours.

10. La déviance est entendue ici au sens qu'en propose Becker, à savoir une " action publiquement disqualifiée [qui est] toujours le résultat des initiatives d'autrui » (Becker, 1985, p. 186).

11. En créole : «Je n'ai pas d'argent ».

12. La «contrainte extérieure » s'oppose à l'«autocontrainte», la contrainte intégrée par le sujet.

13. Concernant les violences physiques ou morales, il est raisonnable de faire l'hypothèse d'une sous- déclaration des pratiques. Les coups sont déclarés par les personnes que le souvenir amuse ou par les plus jeunes d'entre eux encore fortement marqués par l'expérience. Quand on lui demande comment elle se comporterait, en tant que parent, face à l'exclusion d'un de ses enfants, Céline explique : «Je lui demanderais pourquoi et comment ça s'est passé. Je me fâcherais un peu, mais je ne le punirais pas en lui donnant des coups avec un tuyau par exemple. »

14. Terme créole désignant communément un enfant pénible, usant.

15. Une enquête menée à Nottingham auprès de familles afro-caribéennes dont un enfant a été définitivement exclu de son établissement scolaire décrit le caractère préjudiciable de l'exclusion pour les relations familiales. L'exclusion portant notamment atteinte au sens de la « respectabilité » de la famille, en particulier au sein de la communauté proche (Wright et al., 2005).

16. Concernant ces "nouveaux départs ", l'écart de perception entre les professionnels et les familles est marqué, puisque selon les élèves, c'est dans un cas seulement de notre échantillon que l'exclusion est vécue - pour l'un des deux parents - comme une opportunité de réorientation (en l'occurrence, vers une filière professionnelle dans laquelle l'élève sera plus à même de s'épanouir).

17. En France, à côté des enquêtes statistiques, les travaux de Beaud (2002) et Renahy (200512010) ont analysé l'expérience de cette rupture par les jeunes.

\section{RÉSUMÉS}

Chaque année en France, environ 25000 élèves sont convoqués à des conseils de discipline, lesquels débouchent dans plus de deux tiers des cas sur l'exclusion définitive de l'établissement scolaire. Quelles conséquences ont les exclusions scolaires sur les relations à l'intérieur de la famille? À partir d'entretiens menés avec des individus exclus de leurs établissements, nous proposons d'éclairer les réactions parentales à ce qui apparaît comme une véritable épreuve pour la solidarité familiale. L'exclusion d'un établissement scolaire expose ainsi une partie des élèves aux effets conjugués de la rupture scolaire et de la rupture familiale. 
Each year 25,000 pupils in France, are summoned to attend disciplinary boards. More than two thirds of those disciplinary boards lead to permanent school expulsion. From interviews with expelled individuals, this paper sheds light on the parental reactions to what appears to be a strong test for family solidarity. School expulsion exposes pupils to the combined effects of academic failure and family breakdown.

\section{INDEX}

Mots-clés : conseil de discipline, déscolarisation, relations école-famille, rupture scolaire Keywords : School expulsion, school dropout, school-family relations, school disengagement Introduction

\section{AUTEUR}

\section{MICHEL TONDELLIER}

est Docteur en sociologie, maître de conférences à l'Université des Antilles et de la Guyane. Membre du CRILLASH (EA 4095). Thèmes de recherche : Processus de socialisation dans les départements d'Outre-mer, représentations de l'histoire. Contact : Université des Antilles et de la Guyane, Faculté de Lettres et Sciences Humaines - BP 7207, 97233 Schoelcher Cedex. Courriel : michel.tondellier@martinique.univ-ag.fr 